Pierre Anctil

\title{
L'apport du rabbin Pinchas Hirschprung aux lettres juives canadiennes
}


Les mémoires du rabbin Pinchas Hirschprung, parus à Montréal à la fin de 1944, soulèvent plusieurs enjeux littéraires intéressants que l'auteur de cet article aborde dans le contexte de la vie culturelle yiddish au Canada. Quand Hirschprung arrive dans la ville, à la fin de I94I, il n'est guère prédisposé à s'intéresser de près au courant séculier dominant au sein des lettres juives contemporaines. Talmudiste de renom et pratiquant orthodoxe, il n'a pas été préparé par sa formation strictement religieuse à interagir avec les journalistes politiques et les poètes modernistes qui occupent les postes de commande au sein du journal yiddish de Montréal, le Keneder Adler. Malgré cela, il ne tarde pas à être invité à prendre la plume dans ce quotidien et, à la fin de 1942, il publie les premiers chapitres de son futur ouvrage Fun natsishen yomertol: zikhroynes fun a polit dans les pages de l'Adler. Comment s'est établi ce rapport entre le rabbin Hirschprung et ses nouveaux collègues, et quelle signification faut-il tirer de cette rencontre pour l'évolution des lettres yiddish de Montréal?

The Memoirs of Rabbi Pinchas Hirschprung, published in Montreal at the end of 1944, give rise to a number of interesting issues in the context of Canada's Yiddish cultural life. When Hirschprung finally arrives in Montreal, at the end of I94I, he clearly was not associated with the secular impulse that is dominant in contemporary Jewish letters. A renowned Talmudist and an Orthodox Jew, he had not been prepared by his strict religious upbringing to interact with the political journalists and modernist poets who produced the Montreal Yiddish daily, the Keneder Adler. Nonetheless and despite this, he began to write in that daily and, late in 1942, published the first chapters of his future book Fun natsishen yomertol: zikhroynes fun a polit in the pages of the Adler. How did this relationship between Rabbi Hirschprung and the Montreal Yiddish literati become established? Moreover, what historical significance does this fact have on the evolution of Yiddish belles-lettres in Canada?

À la fin de l'année 194I, le rabbin Pinchas Hirschprung est accueilli à Montréal après un long périple à travers la Russie soviétique et l'Asie qui est décrit avec force détails dans son ouvrage publié en novembre 1944: Fun natsishen yomertol: zikhroynes fun a polet [dans la vallée de larmes des nazis; mémoires d'un réfugié]. En fait, il est attendu dans la ville par une communauté juive qui a tout tenté pour lui ouvrir les portes du Canada et lui permettre de trouver enfin un havre de paix. ${ }^{2}$ Arrivé avec quelques autres jeunes pratiquants orthodoxes échappés de justesse à l'occupation allemande de la Pologne puis à la Lituanie occupée par l'armée soviétique, Hirschprung prend place au sein d'un monde qui est le produit de la grande migration esteuropéenne de 1904-I9I4 et qui a pris forme depuis à peine trois décennies. ${ }^{3}$ En I94I, Montréal compte environ 63,000 personnes d'origine juive, dont presque la totalité sont des immigrants récents et ont le yiddish comme langue maternelle. 
La plupart proviennent des petites villes de l'ancien empire russe et ont reçu en Europe une éducation judaïque traditionnelle qu'ils ont ensuite transposée dans leur pays d'adoption. Il y a à Montréal, de plus, un vaste réseau d'institutions religieuses judaïques et d'organisations communautaires qui reflètent les aspirations des Juifs canadiens, notamment des lieux de culte, une autorité rabbinique reconnue et un conseil chargé de l'application des règles de cacherout: le Va'ad Hair. ${ }^{4}$

Cet attachement général à la tradition mosaïque orthodoxe, malgré des adaptations fréquentes à la modernité, explique en bonne partie pourquoi le rabbin Hirschprung juge utile de prendre pied à Montréal pour tenter de refaire sa vie. Sur place il peut espérer obtenir les appuis financiers et les sympathies agissantes nécessaires à la reconstruction d'un mouvement religieux que la montée de l'extrême droite en Allemagne a presque complètement détruit. On trouve aussi dans la ville un quotidien de langue yiddish qui est à la veille de fêter son trente-cinquième anniversaire et dont le propriétaire, Hirsch Wolofsky, est favorable au maintien de la piété et de l'éducation juive traditionnelle. ${ }^{S}$ À la rédaction du Keneder Adler, Hirschprung peut espérer trouver une oreille favorable à ses ambitions et auprès de ses collègues du rabbinat local une écoute attentive. De plus, Montréal possède un réseau d'écoles judaïques auquel se trouve attaché des intellectuels, des pédagogues et des poètes qui forment le noyau d'un mouvement littéraire d'envergure, vaste réservoir de talents et d'activisme culturel au service de la langue yiddish. En I94I, à n'en pas douter, Montréal offre à Hirschprung un environnement judaïque où il est possible pour lui de reprendre le fil brisé de son existence.

Pour bien comprendre la situation à Montréal, au moment où s'installe le rabbin Hirschprung, il peut être utile de consulter le dictionnaire biographique publié par Haim-Leib Fuks en I980, intitulé: Hundert yor Yidishe un Hebreyishe literatur in Kanade [Cent ans de littérature yiddish et hébraïque au Canada]. ${ }^{6}$ Dans cet ouvrage paru à Montréal, l'auteur recense les contributions de 425 écrivains, journalistes, traducteurs et enseignants qui ont produit au pays une œuvre en langue yiddish ou en langue hébraïque, dont une partie non négligeable étaient des adhérents à l'orthodoxie religieuse. Près de $40 \%$ de ces auteurs avaient immigré au Canada juste avant la Première Guerre mondiale et $33 \%$ entre les deux guerres; c'est-à-dire plus de 300 individus dont on peut retracer une contribution aux lettres juives canadiennes dans une langue non officielle. Une étude attentive des données de Fuks nous permet d'ailleurs de constater que les années 1905, I913 et I92I avaient été les plus favorables à la venue au Canada de littérateurs d'origine est-européenne, avec respectivement 16 , 2I et I7 arrivées. En I94I, malgré le conflit qui faisait rage en Europe et les difficultés très grandes qui se dressaient sur le chemin des possibles immigrants, pas moins de huit écrivains de langue yiddish avaient pu s'etablir au pays, dont le très prolifique Melech Ravitch, futur collaborateur à la page littéraire du Keneder Adler et vedette incontestée de la scène culturelle polonaise juive d'avant-guerre. 
À cette époque, trois genres littéraires retiennent particulièrement l'attention des lettrés yiddishophones de Montréal: la poésie, la chronique historique et l'essai politique. Cela tient d'une part à ce que les écrits de ce format trouvent facilement place depuis 1907 dans les pages du Keneder Adler, longtemps le seul débouché viable de la littérature yiddish dans la ville, et de l'autre à ce qu'il s'agit de formes prisées au sein de l'empire russe et auxquelles les Juifs est-européens étaient très sensibles. Un premier recueil de poésie paraît d'ailleurs à Montréal en I918 sous la plume de JacobIsaac Segal: Fun mayn velt [de mon univers], un jeune immigrant arrivé de l'Ukraine en 1910. $\mathrm{Au}$ moment où le rabbin Hirschprung s'installe dans la ville, il existe sur le Plateau Mont-Royal deux écoles littéraires juives distinctes - une première qui exalte l'idéal lyrique et la quête individuelle de la beauté, puis une deuxième dont la caractéristique principale est de promouvoir la justice sociale et l'émancipation du prolétariat des usines de confection. Quand Hirschprung s'installe à Montréal, au moins une centaine d'auteurs se sont déjà fait connaitre du public de langue yiddish dans la ville, dont beaucoup sont des journalistes et des publicistes attachés à différents courants de pensée politique. Souvent ils ressentent une certaine nostalgie de la vie religieuse traditionnelle en Europe de l'Est, ce qui les rapproche d'autant de leurs lecteurs qui souffrent du même penchant. Plusieurs d'entre eux, malgré leur appartenance déclarée au modernisme nord-américain et aux mouvements artistiques d'avant-garde, ont connu au cours de leur enfance les pratiques du judaïsme est-européen et se sentent tout à fait à l'aise d'y faire référence dans leurs œuvres littéraires.

En plus de ces chantres de l'émancipation et du libre arbitre individuel, qui forment la majorité du courant littéraire de langue yiddish à Montréal entre les deux guerres, la ville accueille également des tenants de l'orthodoxie religieuse qui s'expriment eux aussi dans les organes de presse juifs. Le Keneder Adler, qui demeure tout au long de cette période le seul quotidien yiddishophone à paraître dans la métropole québécoise, ne peut se permettre en effet d'ignorer ceux parmi ses lecteurs qui sont attachés à une interprétation strictement religieuse de la judéité. Il accueille ainsi dans ses pages des journalistes d'orientation plus traditionnelle qui reflètent les savoirs judaïques anciens ou discutent le contenu des interprétations rabbiniques classiques. C'est le cas par exemple de Haim Kruger, arrivé à Montréal en 1907, et qui tient pendant des années dans l'Adler une chronique destinée à un public pieux. ${ }^{8}$ Un autodidacte formé dans l'Ancien Monde, Kruger s'intéresse par exemple à l'œuvre du rabbin Yehuda Halevi, à Maimonide, à la philosophie d'inspiration juive et au rabbin Saadia Gaon. D'autres grands esprits associés à l'orthodoxie se joignent à Kruger et prennent la plume régulièrement dans la presse locale, dont le rabbin Zvi-Hirsch Cohen, une des figures dominantes du rabbinat mithnagdique à Montréal depuis le tournant du XX ${ }^{e}$ siècle. ${ }^{9}$ Dans la notice biographique qu'il publie à son sujet en 1980 , Haim-Leib Fuks tient à préciser que Cohen était très actif dans le journalisme: « Il a 
aussi collaboré pendant de nombreuses années au Keneder Adler, publiant à chaque fois que l'occasion lui en était donnée des éditoriaux et des articles concernant les grands enjeux internes ou externes dont était saisi le judaïsme canadien...ce quotidien était pour lui une tribune afin de propager sa parole $»^{\text {10 }}$

D'autres Juifs pieux, et pas des moindres, se sont même carrément lancés dans une carrière littéraire à Montréal, profitant de leur notoriété ou de leurs talents de propagandistes pour publier sur le marché canadien des œuvres de fiction ou des études savantes. Grand érudit et cabaliste d'importance, Yudl Rosenberg avait déjà entrepris en Pologne avant son immigration la rédaction d'œuvres de fiction, dont en 1909 un opuscule décrivant les réalisations légendaires du Maharal de Prague: Niflaot Maharal mi-Prag im ha-Golem [les accomplissements merveilleux du Maharal de Prague avec le golem]." L'ouvrage a connu une diffusion extraordinaire et a même influencé l'œuvre publiée en I92I à New York par le poète moderniste H. Leivick: Der Goylem, a dramatishe poeme in akht bilder [le golem, poème dramatique en huit scènes] et diverses interprétations cinématographiques ultérieures. Une fois installé au Canada en 1913, le rabbin Rosenberg a continué à faire paraitre des tracts religieux à caractère moralisateur. Il a aussi entrepris d'importants travaux de traduction et d'analyse littéraire portant sur la Cabale, dont une partie a paru à Montréal sous forme de volume entre 1924 et 1930. Arrivé plus tardivement à Montréal en 1938, Abraham Shtern a publié dans sa ville d'adoption des études originales du Talmud et de certains versets de la Bible. Il a aussi rendu publique sa correspondance en yiddish et en hébreu, incluant des commentaires sur la Torah, le hassidisme, la Cabale et le folklore traditionnel est-européen. En I953, peu avant sa mort, il publiait à Montréal un dernier volume écrit dans une langue populaire et dédié aux livres saints du judaïsme, à la pensée rabbinique et à Maïmonide, intitulé: Sefer houtim meshoulashim [le livre des ficelles croisées]. Signe que la littérature juive d'inspiration moderne était associée de très près à la tradition judaïque d'expression religieuse, quatre de ses fils ont connu à Montréal des carrières littéraires et journalistiques éclatantes: Yekhiel Shtern, Sholem Shtern, Ish Yair, ${ }^{12}$ et Yaakov Zipper. ${ }^{13}$

Cette ouverture de la presse juive montréalaise à diverses tendances idéologiques et interprétations religieuses en apparence irréconciliables constitue une exception sur le continent nord-américain où, le plus souvent, un journal à grand tirage était associé de près à un courant politique donné. À New York en particulier, une ville où paraissaient plusieurs quotidiens de langue yiddish simultanément, chaque titre conservait une originalité par rapport aux autres et attirait un lectorat bien identifié. Le Morgen Zhurnal [le quotidien du matin], lancé en I9oI, était parcouru par un public surtout attaché à l'orthodoxie religieuse et plutôt conservateur, tandis que le Forverts [en avant] cultivait depuis 1897 des sympathies socialistes. À l'autre extrémité du spectre, le Fraye Arbeyter Shtime [la voix libre des travailleurs] reflétait depuis I89o les thèses politiques anarchistes et le Morgen Frayhayt [la liberté matinale], fondé en I922, 
celles du Parti communiste. Le Keneder Adler, au contraire, avait l'habitude de réunir plusieurs de ces tendances et il cheminait généralement au centre de l'échiquier.

L'Adler ressemblait en fait plutôt au Yidisher Zhurnal [le journal juif] de Toronto, au Yidishe Vort [la parole juive] de Winnipeg ou au Yidishe Velt [le monde juif] de Cleveland, Ohio; toutes des villes où il nétait pas possible de publier plus d'un journal de langue yiddish. Wolofsky, le fondateur de l'Adler, accueillait les revendications ouvrières, mais en se gardant bien au même moment de repousser les adeptes d'un judaïsme plus conservateur. Le journal montréalais savait aussi faire la part belle aux sionistes tout en maintenant ses entrées auprès des yiddishistes, des partisans du nationalisme diasporique et des assimilationnistes. L'Adler, par exemple, encourageait la production littéraire locale en langue yiddish, qui était le plus souvent d'inspiration séculière et moderniste; mais le quotidien n'hésitait pas non plus à rapporter les faits et gestes des rabbins réputés qui vivaient à Montréal ou qui étaient de passage dans la ville. Ce va-et-vient convenait mieux à un public plus restreint sur le plan du nombre et qui n'avait pas de réticence à glaner à droite et à gauche de l'information visant à l'origine des cibles très différentes. Le même parti pris d'universalité était aussi mis en évidence dans d'autres organisations communautaires qui desservaient à Montréal une clientèle immigrante récente, dont la Bibliothèque publique juive qui professait au moment de sa fondation en I9I4 n'avoir aucune intention de soutenir une orientation politique en particulier. ${ }^{14}$

Comme le montre très bien l'ouvrage de Fuks cité plus haut, cette tolérance de bon aloi tenait aussi à ce que la majorité des Juifs est-européens qui étaient immigrés à Montréal au début du $\mathrm{XX}^{e}$ siècle avaient déjà fait l'expérience, dans leur parcours individuel, d'une nécessaire réconciliation entre l'orthodoxie religieuse et la modernité culturelle.5 Presque tous les écrivains, journalistes et enseignants qui sont mentionnés dans le dictionnaire biographique de Fuks avaient été éduqués au cours de leur enfance dans des khadorim, des talmud torot ou des yeshivot, puis avaient ensuite été séduits par l'appel de la haskalah et de l'émancipation politique juive. ${ }^{16}$ Partis de petites villes de l'empire russe, ou de la Pologne nationaliste de l'entredeux-guerres, ils avaient en quelque sorte grandi dans un univers de signification à double visage et côtoyés à la fois le monde rabbinique d'autrefois et les avancées les plus audacieuses de la rationalité scientifique. Même le rabbin Hirschprung ne fait pas vraiment exception à cette règle puis qu'il cite dans son ouvrage plusieurs courants politiques et penseurs de gauche qu'il semble avoir lus attentivement, dont Marx et Lénine. Il fait aussi référence au moment de traverser la frontière soviétique en Pologne à Tolstoï, Dostoïevski et Gorki. Plusieurs auteurs mentionnés dans le dictionnaire biographique de Fuks - sinon la majorité - et à commencer par Fuks lui-même, s'étaient en quelque sorte « convertis " aux nouveaux modes de pensée avant même d'avoir quitté leur shtetl natal, et avaient fait profession de foi envers les idées politiques nouvelles alors qu'ils fréquentaient encore la synagogue ou les 
maisons de prières traditionnelles. Dans la notice le concernant, l'auteur de Hundert yor Yidishe un Hebreyishe literatur in Kanade confesse appartenir à une famille de Lodz «comptant plusieurs rabbins hassidiques » et être le descendant « du gaon et tsadik Avremele Tshernover $»{ }^{17}$ Fuks ne se fait pas de scrupules non plus de déclarer avoir étudié dans plusieurs académies talmudiques au cours de son enfance et avoir obtenu l'autorisation traditionnelle de pratiquer le métier de rabbin. Comme plusieurs de ses collègues littéraires montréalais, Fuks naviguait avec aisance entre les enseignements religieux traditionnels et l'univers des poètes introspectifs new-yorkais.

Pendant que se développait à Montréal une écriture yiddish moderniste et profondément ancrée dans le paysage émotionnel intérieur de quelques poètes dévoués à leur art, des événements menaçants prenaient forme en Europe qui bouleversait la quiétude d'une population adossée depuis plusieurs décennies aux pentes du mont Royal. En Allemagne et dans d'autres pays de l'ancien continent, de nouvelles persécutions anti-juives s'élevaient pour revêtir une ampleur inconnue jusque-là dans l'histoire contemporaine. En l'espace de quelques mois, à la fin des années trente, une série d'incidents et d'attaques, répercutés par la presse partout sur la planète, faisaient craindre que de graves tourments soient à la veille de s'abattre sur le judaïsme est-européen. La situation s'assombrit encore au lendemain de Kristallnacht, puis une fois déclenchée l'invasion de la Pologne par les forces allemandes en septembre 1939. Un brouillard sinistre descend alors sur les populations juives tombées entre les mains des nazis, puis quelques mois plus tard sur les communautés situées plus à l'est dans les pays baltes, en Ukraine et en Russie. Sur le coup, l'ampleur des opérations militaires, la censure imposée par l'occupation et les difficultés de communication rendent opaques les intentions des forces allemandes vis-à-vis des Juifs et insondable leur modus operandi à plus long terme. La distance géographique, le manque d'information crédible et le fait d'avoir vécu au Canada souvent depuis plusieurs années rendent plus impénétrable aux Juifs montréalais le nouveau contexte européen qui se dessine après 1939. Ni victimes ni témoins oculaires de la catastrophe qui prend forme, les yiddishophones canadiens abordent la question de l'occupation du territoire polonais d'un point de vue entièrement différent de leurs coreligionnaires est-européens. Pendant un certain temps, il n'existe ni en Allemagne ni sur place dans les zones occupées de descriptions fiables des méthodes nazies ou de l'ampleur des persécutions antisémites. En particulier, les dirigeants communautaires montréalais sont privés de contacts soutenus et organisés avec la résistance juive ou avec les populations qui sont détenues dans des ghettos réservés exclusivement aux Juifs.

Puis des renseignements commencent à filtrer à propos de massacres systématiques ou de la disparition vers des destinations inconnues d'importants groupes retenus pendant un certain temps dans des centres de transit. Ces données parviennent au Canada via des organismes internationaux neutres, comme la Croix-Rouge et les 
délégations de pays non-belligérants, ou via des mouvements de résistance juifs et non-juifs situés dans les régions occupées par les nazis. Des communications diplomatiques font aussi état, quelques mois après l'invasion de l'URSS, de plans à grande échelle visant, à exploiter, à déplacer ou à éliminer des centaines de milliers de personnes, mais sans que des détails précis n'émergent. ${ }^{18}$ Pour l'essentiel, jusqu'à la fin de la guerre, les dirigeants juifs canadiens en étaient réduits à spéculer sur l'ampleur réelle des exactions, sur les méthodes précises employées par l'occupant et sur les conséquences à court terme de ces persécutions sur les communautés visées. Des témoignages personnels et des récits individuels sont certainement parvenus avant 1943 à des organes de presse yiddish à Montréal, à Toronto ou à Winnipeg, présentés par des gens qui avaient fui l'envahisseur ou s'étaient échappés pendant des transports. Mais ces descriptions formaient un portrait très partiel des événements en cours et étaient souvent reproduites avec plusieurs mois de retard dans des circonstances invérifiables. Les Juifs canadiens en étaient donc réduits le plus souvent à des impressions troubles et à des prémonitions vagues quant à la situation en Europe, ce qui produisait un sentiment d'angoisse et de souffrance bien réel, alimenté par le caractère insondable des témoignages obtenus jusque-là. Rien ne filtrait encore qui puisse donner forme et corps à un processus génocidaire présumé et innommable. Même la visite à Montréal en septembre 1943 de Solomon Mikhoels et de Itsik Fefer, deux envoyés du Comité antifasciste juif d'Union soviétique, ne réussit pas vraiment à lever le voile sur les événements qui se déroulaient dans les régions occupées de l'URSS. ${ }^{19}$

Le sens viscéral de perte et de désorientation profonde qui traverse cette période de l'histoire juive montréalaise, et le désir de mieux informer le public de langue yiddish sur les persécutions en cours, pourraient avoir convaincu le rabbin Hirschprung de tenir la plume pour raconter sa version personnelle des faits. La décision d'écrire et de témoigner n'avait pas dû être prise à la légère, car le projet contrevenait à la vocation solennelle déclarée du jeune talmudiste de servir avant tout l'étude des textes saints et la dissémination des savoirs religieux. Après tout, qu'y avait-il de particulièrement édifiant à faire le récit en direct des brutalités nazies telles que subies par la communauté juive de Dukla; ou celui de la fuite des jeunes étudiants des académies talmudiques à travers le Transsibérien jusqu'à Shanghai, puis leur embarquement à destination d'un port de mer américain, celui de San Francisco. Très peu d'individus étaient sortis intacts en $194 \mathrm{I}$ « de la vallée de larmes des nazis, » selon l'expression maintenant consacrée de Hirschprung, et encore moins qui savaient comment transmettre un portrait fidèle de la situation au-delà des expériences immédiates vécues par les victimes sur le terrain. L’affaire n'allait pas de soi, d'autant plus que le public canadien - même juif - risquait de buter dès le départ sur le caractère nettement invraisemblable à première vue du projet nazi d'éradication des populations juives européennes. Comme l'avaient rapidement constaté pendant la guerre les dirigeants du Congrès juif canadien, pour des lecteurs habitués au 
libéralisme d'inspiration britannique et modérés sur le plan politique, le récit des persécutions juives prenait rapidement des allures de fabulation propagandiste.

L'incertitude générale, le manque de renseignements validés et l'absence de précédents historiques allaient vite contraindre le rabbin Hirschprung à s'en tenir à un style d'écriture direct et libre de tout recours à l'hyperbole. L'allure prosaïque du récit, son caractère avant tout descriptif et l'actualité du sujet traité contredisaient cependant entièrement l'éducation que le jeune auteur avait reçue à la yeshiva. En d'autres temps, il aurait été perçu à proprement parler comme scandaleux qu'un étudiant des meilleures institutions judaïques polonaises - et l'un des plus doués de surcroît - détourne le regard des textes saints pour emprunter un ton journalistique. C'est pourtant ce que Hirschprung s'employa à faire à un certain moment au cours de l'année 1942, une fois régularisée sa situation de réfugié en temps de guerre. Il fallait que le désir d'informer et de sensibiliser soit très puissant pour que Hirschprung consente momentanément à interrompre ses tâches rabbiniques et communautaires, pour plonger dans la rédaction d'un ouvrage détaché des interprétations exégétiques. ${ }^{20}$ Or, le natif de Dukla détenait des informations privilégiées et avait été témoin de nombreuses scènes dont les Juifs montréalais ignoraient tout ou ne pouvaient qu'imaginer vaguement. Il était même entré en contact au début du conflit militaire avec des officiers allemands en Pologne et avait dû négocier des visas de sortie soviétiques à un moment où il était encore possible, pendant une brève période en août 1940, de s'échapper de la Lituanie indépendante. Pour ses lecteurs canadiens, son récit revêtait une grande importance par ce qu'il révélait des forces en présence au cours de l'invasion allemande de la Pologne, et du traitement des populations juives devenues prisonnières de circonstances très inquiétantes. Le témoignage du jeune talmudiste échappé de "l'enfer » prenait immédiatement un relief exceptionnel dans une ville où ne parvenaient que de loin en loin les échos d'une tragédie effroyable, mais dont nul ne parvenait à fixer les contours précisément. Enfin quelqu'un arrivait à la fin de i94I qui pouvait prendre la parole alors que partout régnait un silence de plomb, quelqu'un dont la réputation était inattaquable sur le plan moral et qui offrait de fortes garanties de rectitude spirituelle. C'était inespéré.

Nous ne sommes toujours pas en mesure aujourd'hui de savoir exactement comment le rabbin Hirschprung résolut son dilemme moral relativement au fait d'écrire pour un organe de presse sans vocation religieuse particulière, ni s'il consentit sans réticence à aborder des sujets séculiers. Nous pouvons toutefois dater assez précisément le début de sa collaboration avec le Keneder Adler, prélude à la publication de son ouvrage autobiographique de novembre 1944. Le 2 octobre 1942, Hirschprung fait paraître dans l'Adler une discussion de nature talmudique intitulée: « Talmud Bavli, Talmud Yerushalmi un 'Talmud Kvibeki' [le Talmud de Babylone, le Talmud de Jérusalem et le 'Talmud de Québec']. »C'était suite à la parution dans l'Action catholique, sous la plume d'un prêtre franciscain, d'une série de trois articles à saveur doctrinale 
portant le titre de « La question juive. $»^{2 \mathrm{I}}$ Dans ces textes, l'auteur décrivait le Talmud comme un document perfide avalisant l'exploitation commerciale des chrétiens par les Juifs. L'accusation n'était guère originale ni le ton adopté différent de celui emprunté par d'autres détracteurs catholiques du judaïsme à différentes époques. Ce qui était inhabituel, c'est qu'un grand érudit du judaïsme se laisse entraîner dans une polémique - en yiddish - avec un homme d'Église et relève un certain nombre d'errements dans le raisonnement théologique proposé aux lecteurs d'un quotidien de Québec, l'Action catholique. La contribution de Hirschprung avait été précédée à la fin du mois de septembre, dans les pages de l'Adler, de quatre articles de l'un des journalistes et chroniqueurs les plus estimés de la presse yiddish canadienne, Benjamin-G. Sack. Dans ces textes à l'allure polémique, Sack relevait avec verve l'attaque essuyée par les Juifs dans l'Action catholique en mai et en juin de la même année, et pavait la voie à l'intervention Hirschprung. Sack, qui lisait couramment le français et était un résident de Montréal depuis une trentaine d'années, s'insurgeait dans « A vilde oygenglotserishe atake af Yiden [une attaque sauvage et délibérée contre les Juifs] $\gg^{22}$ du ton adopté par les contempteurs catholiques du judaïsme et tentait de corriger toujours en yiddish - l'impression négative laissée par les écrits du père Bonaventure Péloquin. ${ }^{23}$ Sans doute peu à l'aise dans les complexités du raisonnement talmudique, et toujours dans le but d'appuyer son propos, Sack et les rédacteurs de l'Adler avaient jugé utile quelques jours plus tard de faire appel à Hirschprung pour donner plus d'autorité à leur dénonciation des préjugés catholiques. Il y a peu de chances en effet que les arguments antisémites du père Péloquin soient parvenus d'eux-mêmes aux oreilles d'un Hirschprung plongé dans son propre travail de talmudiste.

Le ton de l'article du 2 octobre 1942, et l'à-propos de sa publication au lendemain du reportage de Sack, nous laisse croire que le rabbin Hirschprung avait déjà pris contact à cette date avec le personnel du journal ou à tout le moins avec son propriétaire, Hirsch Wolofsky. L'hypothèse semble d'autant plus probable que Wolofsky était un des grands leaders communautaires du Montréal juif et qu'il ne répugnait pas à fréquenter assidûment les autorités rabbiniques de la ville. Il avait été un des principaux promoteurs en 1923 de la fondation du Va'ad Hair, l'organisme chargé de la gestion de la certification cachère à Montréal, et était lié d'amitié depuis longtemps avec le rabbin Zvi-Hirsch Cohen. Nous savons également que les visas canadiens qui avaient permis l'entrée des étudiants talmudiques bloqués à Shanghai en 194I avaient été négociés à Ottawa par les dirigeants juifs de Montréal, dont l'omniprésent Wolofsky. ${ }^{24}$ Le texte publié par Hirschprung, le 2 octobre 1942, a aussi la particularité d'avoir été précédé d'une courte préface, signée de la rédaction du Keneder Adler, et qui introduit pour la première fois le réputé talmudiste au lectorat du journal. Grâce à ces quelques lignes, nous pouvons mieux comprendre comment était perçue la contribution de Hirschprung à la presse yiddish de Montréal et quelle position le savant occupait dans l'espace culturel juif canadien. Pour l'essentiel, rappelait le personnel de l'Adler, l'on faisait appel à Hirschprung pour clarifier des questions doc- 
trinales judaïques et non pas pour présenter un positionnement politique ou pour livrer des impressions sous une forme littéraire. Le rabbin, prévenait la direction du journal, appartenait à un autre univers de signification que celui du journalisme et ne venait pas par ses écrits défendre les revendications légitimes de la communauté juive sur la scène québécoise ou face à l'Église catholique:

Le respecté auteur de cet article est le nouveau rabbin de la synagogue Adath Yeshurun-Hadrath Kodesh. Il est considéré comme l'un des plus grands talmudistes. Sa mémoire phénoménale est extraordinaire. Si vous lui posez une question au sujet d'un aphorisme du Talmud, il vous répondra dans quel traité, dans quel chapitre et dans quel feuillet se trouve ce passage. Le présent article porte sur l'attaque d'un prêtre de Québec et sa supposée citation du Talmud reproduite dans le journal «pieux» de Québec, l'Action catholique. Quiconque veut connaître la vérité au sujet du rapport du Talmud à cette abomination doit lire cet important article d'un véritable expert en littérature talmudique. ${ }^{25}$

Les intentions du Keneder Adler se précisent quand le journal commence le 25 décembre 1942 à publier par segments les mémoires du rabbin Hirschprung sous le titre: «Der Eyropeyisher gehenem; zikhroynes fun a polet [l'enfer européen; mémoires d'un réfugié]. " Il est fort probable que le texte de Hirschprung existait déjà au complet avant que sa parution ne débute dans le journal et qu'il n'a pas été écrit au fil des semaines pour satisfaire le lectorat de l'Adler. Aucune mise en contexte n'entoure la parution des épisodes, qui sont reproduits en haut à gauche de la page 5 , si ce n'est une photo du grand-père de l'auteur, le rabbin David-Zvi Sehmann. La série ne connaît cependant que onze livraisons et se termine abruptement le 26 janvier 1943, sans aucune explication ni commentaire de la part de la rédaction. Pendant cette courte période paraissent les neuf premiers chapitres du futur livre, soit depuis le chapitre « Mayn shtetele Dukla [ma bourgade Dukla] » jusqu'à « Der araynmarsh fun der Daytshisher armey in Dukla [l'entrée de l'armée allemande à Dukla]. » Cela équivaut en gros aux quarante premières pages du récit, qui en comptera 249 une fois publié en totalité à la fin de i944. Quand finalement la publication des mémoires de Hirschprung reprend le 3 mai 1944 dans le Keneder Adler, après un long hiatus de plusieurs mois, son récit est maintenant présenté sous le titre de Fun natsishen yomertol: zikhroynes fun a polet [dans la vallée de larmes des nazis (mémoires d'un réfugié)]. Craignant de dénaturer l'œuvre, le journal reproduit le 3 et le 4 mai le premier chapitre du récit, intitulé: « Mayn shtetele Dukla, » puis offre le 5 mai un résumé des huit épisodes déjà publiés du 29 décembre 1942 au 26 janvier 1943. La matière inédite reprend le 6 mai 1944 avec le chapitre intitulé: « Daytshe soldaten 'koyfen' oys di gesheftn fun gants Dukla [les soldats allemands 'font l'acquisition' de tous les commerces de Dukla. " Le texte apparaît à gauche en une seule colonne sur la quatrième page du quotidien et s'étend par sections jusqu'au is août i944. Publié 
face à léditorial rédigé par la direction, il occupe une place d'honneur dans le journal et court sur plus de trois mois, jour après jour, sans interruption dans le texte ou mention de chapitres.

À n'en pas douter, Wolofsky et Israël Rabinovitch, le rédacteur en chef, accordent au rabbin et à son propos une valeur certaine et tentent d'attirer l'attention des lecteurs sur sa parution. Une note insérée par la rédaction le 3 mai 1944 nous apprend que la publication de l'ouvrage avait été suspendue lors d'une première tentative à la fin de l'année 1942, pour des raisons qui ne sont pas précisées et malgré le succès initial obtenu: « Tsulib tamim kmousim - tamim vos konen nit farefentlikh veren - hot harav Hirschprung gemuzt tsaytvaylik opshtelen dos forzetsn fun der serye. $»^{26}$ Cela pourrait signifier que des scrupules d'ordre moral avaient empêché l'auteur de livrer la substance de son témoignage et qu'il avait peut-être dû essuyer des critiques dans certains milieux de pratique religieuse orthodoxe, outrés du caractère éminemment prosaïque de l'œuvre. Il est fort peu probable que de telles réticences soient venues de la part de Wolofsky et de son équipe, qui escomptaient plutôt soulever l'intérêt de leurs lecteurs avec une parution organisée par sections. On peut aussi supposer que Fun natsishen yomertol contenait des informations privilégiées susceptibles de nuire à des personnes restées en Europe ou qui risquaient de mettre en péril la vie de ceux qui avaient soutenu les étudiants talmudistes dans leur fuite, dont le professeur Abram Kotsuji, un professeur de théologie à l'Université de Tokyo et un spécialiste du judaïsme. La photographie de ce dernier, en compagnie du rabbin Abraham-Mordecai Herschberg, paraissait d'ailleurs dans le dernier épisode publié le I5 août 1944 dans l'Adler. Il est possible aussi que la censure militaire ait jugé que la mention du Japon sous un jour favorable, un pays auquel le Canada avait déclaré la guerre le 7 décembre 194I, ait été contraire à l'intérêt des forces Alliées. Quoi qu'il en soit, la rédaction du Keneder Adler, à en juger par la note du 3 mai 1944, était enchantée de pouvoir enfin faire paraitre le récit du rabbin dans son intégrité:

À la fin de 1942, le rabbin Hirschprung a commencé à faire paraître dans le Keneder Adler une série intitulée: « Mémoires d'un réfugié, » qui a été suivie avec beaucoup d'intérêt par nos lecteurs. [...] Nous sommes heureux d'annoncer que cet intéressant récit va être publié à partir de maintenant sans interruption. Afin que la série ne perde pas de sa cohérence, nous débutons le premier chapitre avec un résumé des chapitres déjà publiés. ${ }^{27}$

On peut mieux saisir le caractère particulier de la publication des mémoires du rabbin quand on prend le temps d'analyser quels types de feuilletons étaient proposés dans les pages du Keneder Adler au cours des années 1942 à I944. À cette époque, tout journal populaire qui se respectait - même ceux de langue yiddish - avait le devoir de reproduire à l'intention de son public des textes de fiction qui paraissaient sur de longues périodes et qui offraient un espace de divertissement clairement identifié. 
L'Adler ne faisait pas exception à cette règle, même si par ailleurs certaines de ses chroniques hebdomadaires et de ses reportages pouvaient à l'occasion revêtir un caractère nettement élitiste et littéraire. Pour attirer l'attention des plus distraits et fidéliser les lecteurs irréguliers, ces feuilletons devaient être offerts sur une base quasi quotidienne et apparaître dans la partie supérieure droite d'une page sous un titre accrocheur. À l'automne I942, par exemple, l'Adler proposait « Der royter hon [le coq rouge], » un texte de Mark Sardel présenté comme « un roman passionnant sur la vie juive en Amérique. » Les pages du journal contenaient aussi cette saison-là «Af yener zayt taykh [de ce côté de la rivière] $»^{28}$ d'Aron Feldman, un texte décrit comme « a realistisher roman fun Yidishe leben in Eyrope un Amerike [un roman réaliste de la vie juive en Europe et en Amérique]. » On pouvait aussi lire en même temps dans l'Adler « In shpigl fun leben [dans le miroir de la vie] » du docteur Ratgeber ${ }^{29}$ et « Lomir ze khotsh gedenken [rappelons-nous tout de même d'eux] » de Melekh Ravitch, un ensemble de courts textes décrits comme des " portraits intimes et des souvenirs au sujet de figures juives connues de Pologne. ».3 À part « In shpigl fun leben » qui poursuit sur sa lancée, l'année I943 apporte aux lecteurs « Likhtike geshtaltn [Personnalités remarquables] » du Dr Yud Lamed Shnitzer, un texte décrit comme «un roman historique, » et " Mayn lebens rayze [le périple de ma vie] » de Hirsch Wolofsky, qui sont les mémoires du fondateur de l'Adler sur plus d'un demi-siècle. ${ }^{3 \mathrm{~A}} \mathrm{~A}$ l'automne 1943 un nouveau venu fait son apparition, « Der vagabond [le vagabond] » d'Aron Feldman, décrit comme un «roman écrit spécialement pour l'Adler, » puis au début de 1944 " Me hot gelebt mit bitokhn [nous avons vécu dans la confiance] » de Berl Boym, présenté avec sous-titre: «Zikhroynes fun der alter haym [souvenirs de l'Ancien Monde] ». En avril, juste avant que ne débute la série consacrée au rabbin Hirschprung, en plus de «In shpigl fun leben, » qui continue sa longue course, paraît pour la première fois « Der Yidisher held fun Poylen [le héros juif de Pologne] » d'Itshe V. Biderman, aussi un « roman historique. » Essentiellement, il s'agit de courts récits visant à distraire l'auditoire de l'Adler, surtout en fait des textes de fiction qui présentent une perception bienveillante et nostalgique de la vie juive outre-Atlantique d'un point de vue historique.

Comme on peut le constater, nous sommes loin ici des préoccupations premières $\mathrm{du}$ rabbin Hirschprung et de son style d'écriture prosaïque consacré à décrire l'arrivée des forces allemandes dans son patelin d'origine, puis sa fuite à travers l'URSS. De fait, même au plus fort de la Seconde Guerre mondiale, l'Adler s'intéresse très peu dans ses feuilletons aux événements qui se déroulent alors en Europe de l'Est. Il en va tout autrement par contre des chroniqueurs et journalistes les plus expérimentés du journal. Le poète Jacob-Isaac Segal, par exemple, qui n'est certainement pas un écrivain très porté sur l'actualité politique, aborde le contexte des agressions nazies dans plusieurs courts essais publiés dans l'Adler au cours des années 1943 et 1944. Sur un ton tragique, Segal s'interroge à plusieurs reprises sur la survie du judaïsme est-européen et sur le sort des populations emportées dans la tourmente..$^{32}$ Toutefois, 
à la différence de Hirschprung, aucun des écrivains canadiens qui s'exprimaient dans l'Adler n'avait été un témoin oculaire des violences extrêmes qui s'exerçaient contre les Juifs en Europe occupée, pas même Melech Ravitch qui avait quitté la Pologne en 1934. Leur sentiment d'outrage et de révolte était celui de personnes qui vivaient depuis longtemps à l'abri des pires abus, entourés des protections légales que leur conférait le régime parlementaire canadien. Les auteurs yiddishophones montréalais, dont certains avaient immigré avant la Première Guerre mondiale, s'étaient peu à peu éloignés avec le passage du temps des réalités est-européennes les plus courantes. Si leur douleur était bien palpable et leur souffrance aiguë, elles ne se comparaient en rien à celle que ressentaient les individus qui avaient été témoins jour après jour de scènes bouleversantes et de situations cataclysmiques.

Nous avons deux autres points de référence importants pour juger du caractère exceptionnel de la publication des mémoires du rabbin Hirschprung dans l'Adler. Pour les écrivains montréalais de langue yiddish et dans les différents cercles culturels apparus au début des années vingt autour de la Bibliothèque publique juive, l'écriture revêtait un caractère essentiellement moderniste et hautement littéraire. Ce sont au sein de ces différents milieux qu'avaient été recrutés les principaux collaborateurs et journalistes qui œuvraient au quotidien dans le journal de Wolofsky. Pour les adeptes canadiens des écoles littéraires américaines Di Yunge et In Zikh, il ne suffisait pas de rédiger des textes et de faire état de ses sentiments. Encore fallait-il que le résultat se mesure aux œuvres les plus audacieuses publiées dans les différentes langues européennes et qui servaient le plus souvent de modèle aux jeunes talents canadiens de langue yiddish. C'était particulièrement le cas de Melekh Ravitch, coresponsable avec Jacob-Isaac Segal de la chronique hebdomadaire de haute tenue qui a commencé à paraitre dans l'Adler après 1942 sous le titre de « Di literarishe vokh [la semaine littéraire] ». Sur cette page paraissaient régulièrement des critiques inspirées de l'actualité littéraire sur deux continents, de courts essais portant sur certains auteurs en particulier, et des poèmes de première valeur. Or, cette chronique est restée muette au sujet de la parution à la fin de 1944, sous forme de livre, des mémoires de Hirschprung. Nul ne songe dans « Di literarishe vokh » à commenter ou à mentionner la publication d'un ouvrage qui avait dû être fort remarqué à l'époque et qui avait trouvé une place dans les pages de l'Adler pendant plus de trois mois. La raison principale de ce silence est que Fun natsishen yomertol ne se rattachait à aucun courant littéraire particulier et que son auteur ne considérait pas son travail d'écriture comme inspiré par une pulsion artistique. Les mémoires de Hirschprung, rédigés dans une langue simple et descriptive, ne reflétaient aucune volonté d'élever le récit au rang d'une œuvre au sens noble du terme. Contrairement à Chava Rosenfarb, Rokhl Korn ou Mordecai Husid, tous des écrivains arrivés à Montréal après 1948 et qui firent de la Shoah un thème littéraire de première valeur, Hirschprung visait à livrer une représentation factuelle et immédiate des événements dont il avait été témoin. 
Israël Rabinovitch, le rédacteur en chef de l'Adler, ne réagit pas autrement quand il signe la préface du volume paru en novembre i944 aux presses du journal qu'il dirige. Pour lui, Fun natsishen yomertol n'appartient pas à l'univers des lettres yiddish, ni par son style d'écriture ni par sa mention fréquente de l'intervention divine dans les affaires humaines. L'écriture yiddish à Montréal était en effet fortement tributaire d'une vision séculière de l'existence et si certains de ses contributeurs faisaient référence à l'occasion aux pratiques religieuses judaïques; par exemple, Jacob-Isaac Segal, ce n'était pas pour faire état de leur confiance absolue en Dieu ou pour faire étalage de leurs connaissances talmudiques; bien au contraire. ${ }^{33}$ Plusieurs des plus éloquents poètes yiddishophones du Canada avaient reçu dans leur pays d'origine une éducation traditionnelle assez semblable à celle du rabbin Hirschprung, mais ils s'étaient détachés assez tôt dans leur vie de ce profond courant de piété et considéraient l'héritage judaïque - à la manière du grand historien Simon Dubnov comme relevant avant tout à la période moderne de la sphère nationale et culturelle. Interloqué par la forme et le contenu de Fun natsishen yomertol, Rabinovitch concède dans sa préface que l'ouvrage, sans se hisser au sommet de la production littéraire, n'en a pas moins une grande valeur pour un public montréalais privé d'information fiable au sujet du sort réservé aux Juifs pendant la guerre:

Les expériences vécues qu'il nous raconte [Hirschprung] ne sont pas à proprement parler de la « littérature. »Cela relève plutôt de la pure vérité. [...] Je laisse donc le soin aux critiques de juger de la qualité littéraire de ce livre. Pour ce qui me concerne, je me contente d'applaudir à la parution de ce livre en tant que document d'une grande valeur humaine. ${ }^{34}$

Parions que l'œuvre du rabbin Hirschprung n'aurait sans doute pas vu le jour dans l'Adler en d'autres circonstances, ni sous la forme d'un feuilleton et encore moins quelques semaines plus tard comme un livre. Avant la guerre, peu de textes de ce genre avaient paru dans les pages du journal yiddish parce que le témoignage direct et dénué de fioritures littéraires ne correspondait pas au sens esthétique du public yiddishophone montréalais. Cela tenait à ce que les Juifs canadiens cultivaient un certain sens de l'optimisme et de l'hyperbole, et percevaient leur propre société à travers le prisme d'une mobilité socio-économique assez prononcée. Détachés de l'Europe depuis plusieurs décennies, ils conservaient en toute circonstance l'espoir de s'intégrer raisonnablement bien à leur pays et dans des délais assez courts. C'est cet élan optimiste que le deuxième conflit mondial va briser de manière irrémédiable, même si dans les faits l'émancipation politique et sociale des Juifs canadiens continue de progresser au pays après la guerre. En fait, les nouvelles en provenance de l'Europe occupée sont si tragiques après i94I, et signifient un tel bouleversement du rapport de force historique au sein de la diaspora, que toute bribe d'information à ce sujet valait son pesant d'or. Déjà en 1944, le Keneder Adler conçoit qu'il y a un auditoire de grande ampleur pour ce genre de témoignage, même si le récit de Hir- 
schprung - lorsqu'il parait finalement au complet - relate une situation antérieure d'au moins trois ans. Ceux qui lisent ce texte dans l'Adler, tel que paru de mai à août I944, se doutent bien que le martyr des populations juives a terriblement progressé depuis que ces lignes ont été écrites et que la tragédie arrive à son terme. Malgré ce décalage temporel assez prononcé, le récit de Hirschprung demeure au moment de sa parution une référence de premier ordre au sein de la littérature juive canadienne.

Cela tient surtout, comme l'a démontré Franklin Bialystok dans Delayed Impact, , à $^{35}$ ce que les Juifs canadiens, même ceux immigrés dans l'entre-deux-guerres, n'ont pas de point de repère précis pour aborder les événements qui se produisent après l'invasion de l'URSS par les forces allemandes. Les massacres systématiques à grande échelle, les déplacements forcés de population et la ghettoïsation menant à lélimination en masse des Juifs n'ont pas d'équivalent dans l'histoire tsariste, pourtant riche en persécutions antisémites de toutes sortes. La situation est encore plus contrastée en Amérique du Nord où peu de violence s'exerce contre les populations juives. Pour un lectorat qui s'insère dans une démocratie libérale de type britannique, même imparfaite et même hostile à l'immigration des Juifs allemands à la fin des années trente, le récit du rabbin Hirschprung présente une rupture profonde avec ce qui est su et connu. À terme, l'œuvre annonce la destruction du judaïsme est-européen et l'anéantissement des innombrables milieux de vie et communautés dont étaient issus en grande partie les Juifs montréalais, une réalité avec laquelle les lecteurs de Fun natsishen yomertol ne peuvent certes pas se réconcilier facilement. Pour beaucoup de yiddishophones qui vivent à l'ombre du mont Royal, les violences brutales qui s'exercent contre l'univers juif est-européen atteignent des êtres proches et réduisent à néant des localités auxquels sont rattachés des souvenirs personnels encore persistants.

Dans ce contexte, la publication des mémoires du rabbin Hirschprung en 1944 devient un point tournant de l'histoire juive montréalaise; d'abord parce c'est la première fois qu'un témoignage hautement crédible concernant la situation en Pologne et en Russie parvient au lectorat yiddishophone canadien, mais aussi parce que le texte annonce un changement fondamental de paradigme dont les répercussions se font sentir jusqu'à aujourd'hui. Hirschprung ne pouvait être taxé de propagandiste de la cause des Alliés et son jugement était de toute évidence resté inaltéré par la catastrophe qui s'abattait sur les siens. Ce qu'il soumettait au lectorat le lendemain de son arrivée à Montréal revêtait une grande valeur sur le plan moral et éthique. Plus personne ne pouvait douter en 1944 que le judaïsme européen, qui avait donné naissance au XVIII ${ }^{e}$ siècle aux nouveaux établissements juifs de la vallée du SaintLaurent, et avait nourri pendant des décennies leur développement spirituel et culturel, gisait en lambeaux et sombrait maintenant dans le néant. La nouvelle, corroborée par un grand talmudiste et héritier d'une tradition très respectée, de surcroît témoin oculaire du désastre, forçait les Juifs canadiens à une redéfinition très douloureuse 
de leur position dans le judaïsme mondial. Dorénavant, l'Europe ne serait plus la mesure principale et la référence obligée de la grande tradition ashkénaze qui avait dominé la judéité pendant plus d'un millénaire. Des siècles d'histoire, d'introspection religieuse et d'enracinement s'envolaient en fumée sur l'ancien continent, qui ne pourraient plus être reconstitués ou transposés ailleurs. À l'évidence, un basculement subit et imprévisible remettait entre les mains des Juifs nord-américains la responsabilité de perpétuer l'héritage est-européen, et plus lointainement entre les mains des nouvelles colonies agricoles sionistes établies en Palestine sous le mandat britannique.

La catastrophe obligeait aussi les Juifs montréalais à repenser les bases de leur univers culturel en grande partie arrimé à la langue yiddish. La jeune littérature juive canadienne entre autres, qui avait commencé dès le début du siècle à briller dans le firmament des lettres est-européennes, et avait recruté entres les deux guerres de talentueux représentants des milieux culturels russes et polonais, perdait dans l'intervalle une grande partie de son lectorat potentiel. Il était maintenant irréfutable que les milieux de vie et les pratiques religieuses qui avaient inspiré ses auteurs les plus talentueux avaient été annihilés dans le maelstrom du deuxième conflit mondial. Où les poètes et les écrivains yiddishophones canadiens trouveraient-ils la force de poursuivre, quand ce qui les avait inspirés pendant si longtemps avait subitement cessé d'exister ? L'élite intellectuelle et littéraire est-européenne avec laquelle ils avaient partagé leurs premiers pas et échangé de manière soutenue était maintenant disparue ou était dispersée aux quatre coins de l'univers. À tout le moins le massacre des populations juives sonnait le glas de l'esprit d'optimisme et d'ouverture au monde qui avait caractérisé les quarante premières années de la vie culturelle de langue yiddish à Montréal. Lélan des débuts et l'espoir de la jeunesse faisaient maintenant place à des constats désespérants, qu'une nouvelle immigration d'après-guerre composée surtout de survivants de l'Holocauste allait accentuer. Après le rabbin Hirschprung, la description des conditions imposées par les nazis dans les ghettos et les camps de la mort, le souvenir des disparus et la peine ressentie par les rescapés du Troisième Reich prendraient le devant de la scène dans la littérature yiddish canadienne, soit sous la forme de récits descriptifs, soit grâce à la parution d'une poésie éplorée et sombre. Un tournant était pris qui serait décisif dans l'histoire du judaïsme montréalais. 
1

Rabbin Pinchas Hirschprung, Fun natsishen yomertol; zikhroynes fun a polet (Montréal, Eagle Publishing Co., novembre 1944). L'ouvrage est traduit en anglais sous le titre The Vale of Tears par Vivian Felsen (Montréal, la Fondation Azrieli, 2016).

\section{2}

C'est un contexte qui est décrit dans

l'ouvrage d'Israël Medresh, Tsvishen tsvey velt milkhomes, paru à

Montréal en 1964. L'ouvrage est disponible en français sous le titre Le Montréal juif entre les deux guerres (Sillery, Éditions du Septentrion, 2001). Le passage en question est aux pages 194-195.

\section{3}

Voir Pierre Anctil et Ira Robinson (dir.), Les communautés juives de Montréal, histoire et enjeux contemporains (Sillery, les Éditions du Septentrion, 2010).

\section{4}

Steven Lapidus, Orthodoxy in Transition; the Vaad Ha'ir of Montreal in the Twentieth Century (Montréal, Université Concordia, thèse de doctorat en études religieuses, 2011).

\section{5}

David Rome et Pierre Anctil, Through the Eyes of the Eagle: The Early Montreal Yiddish Press 1907-1916 (Montréal, Véhicule Press, 2001). L'ouvrage contient de nombreux extraits de la presse yiddish de Montréal à ses débuts.

\section{6}

Haim-Leib Fuks, Cent ans de littérature yiddish et hébraïque au Canada [Hundert yor Yidishe un Hebreyshe literatur in Kanade], traduit au français par Pierre Anctil (Sillery, Éditions du Septentrion, 2005).

\section{7}

Voir la biographie de Segal rédigées par l'auteur de cet article et parue aux Presses de l'Université Laval en 2012 sous le titre: JacobIsaac Segal (1896-1954); un poète yiddish de Montréal et son milieu.

8

Pour ce sujet en général voir: Ira Robinson, Rabbis and Their Community; Studies in Eastern European Orthodox Rabbinate in Montreal, 1896-1930 (Calgary, University of Calgary Press, 2007).
9

Steven Lapidus, « 'Maggid of Montreal' : Rabbi Hirsch Cohen on the Dilemma of the Canadian Rabbi, » Jewish History, numéro spécial intitulé: "Sermon and Society in the Canadian Jewish Experience, » 23, n² (2009): 179-193.

\section{0}

Haim-Leib Fuks, Cent ans de littérature yiddish et hébraïque au Canada, 138-139.

11

Ira Robinson, « Kabbalist and Community Leader: Rabbi Yudel Rosenberg and the Canadian Jewish Community, " Canadian Jewish Studies / Études juives canadiennes 1 (1993): 41-58. Voir aussi l'oeuvre du rabbin Rosenberg traduite par Curt Leviant sous le titre: The Golem and the Wonderous Deeds of the Maharal of Prague (New Haven, Conn., Yale University Press, 2008).

\section{2}

Nom de plume de Yisroel-Hersh Shtern.

13

Nom de plume de Jacob-Isaac Shtern.

\section{4}

Voir Pierre Anctil, « 'Créée par le peuple et pour le peuple': réflexions sur les origines historiques de la Bibliothèque publique juive de Montréal, „Études juives canadiennes / Canadian Jewish Studies 22 (2014): 32-53.

\section{5}

Voir à ce sujet le témoignage d'Israël Medresh dans Le Montréal juif d'autrefois, [Montreal fun nekhtn], (Sillery, Éditions du Septentrion, 1997). Traduit du yiddish par Pierre Anctil.

\section{6}

Voir aussi le témoignage de Hirsch Hershman dans « 25 yor yidish arbeter bavegung in Montreal, » Unzer Vort [notre parole], Montréal, 23 décembre 1927 au 2 mars 1928; publié sous le titre « À l'occasion des vingt-cinq ans du mouvement ouvrier juif à Montréal, » dans Bulletin du Regroupement des chercheurs en histoire des travailleurs $d u$ Québec 26, n 1 (printemps 2000): 54. Traduit en français par Pierre Anctil.

17

Voir la biographie de Haim-Leib Fuks dans Cent ans de littérature yiddish et hébraïque au Canada, 288-289. 
18

Consulter à ce sujet: David S. Wyman, The Abandonment of the Jews; America and the Holocaust, 1941-45 (New York, Pantheon Books, 1984).

19

Voir le récit de Sholem Shtern à ce sujet dans Nostalgie et tristesse, mémoires littéraires du Montréal yiddish [Shrayber vos ikh hob gekent, memuarn un esayn] (Montréal, les Éditions du Noroît, 2006, p. 163-178). Traduit en français par Pierre Anctil.

\section{0}

Il est vrai que le récit du rabbin Hirschprung contient néanmoins de nombreuses allusions à la pratique judaïque et aux convictions religieuses de l'auteur. On y trouve notamment des références fréquentes à des passages de la Bible, du Talmud et à des prières courantes.

\section{1}

Père Bonaventure Péloquin, o.f.m., « La question juive, " L'Action catholique, 5 mai 1942, 4; «La question juive. Attitude de l'Église au cours des âges, " L'Action catholique, 18 mai 1942, 4; et « La question juive. Attitude de l'Église au cours des âges. Législation ecclésiastique, " L'Action catholique, 2 juin 1942, p. 4.

\section{2}

Benjamin-G. Sack, « A vilde oygenglotserishe atake af Yiden [une attaque sauvage et délibérée contre les Juifs], » Der Keneder Adler, 20, 23, 25 et 30 septembre 1942.

\section{3}

On trouve une courte biographie du père Péloquin dans l'ouvrage publié sous la direction de Jean Hamelin: Les Franciscains au Canada, 1890-1990 (Sillery, Éditions du Septentrion, 1990).

\section{4}

Voir le chapitre intitulé : «Le rabbin Zvi Cohen » dans les mémoires de Hirsch Wolofsky intitulées en français: Mayn Lebens Rayze; un demi-siècle de vie yiddish à Montréal et ailleurs dans le monde [Mayn lebns rayze. Zikhroynes fun iber a halbn yorhundert Yidish lebn in der alter un nayer velt], " Sillery, Éditions du Septentrion, 2000, 344-358. Wolofsky mentionne le rabbin Hirschprung à la page 350.
25

Le rabbin Pinchas Hirschprung, «Talmud Bavli, Talmud Yerushalmi un Talmud Kvibeki [le Talmud de Babylone, le Talmud de Jérusalem et le Talmud de Québec], » Der Keneder Adler, 2 octobre 1942. La citation en yiddish se lit comme suit : « Der khoshever shrayber fun dem artikel iz der nayer rov fun Adath Yeshurun-Hadrath Kodesh shul, velkher vert batrakht als ayner fun di greste talmudisten. Zayn fenomenaler zikorn iz oysergevayntlekh. Vegen velkher memre fun talmud ir zolt im nit fregen, vet er aykh zogen genoy in velkher mesekhte, in velkher peyrek un oyf velkhe daf dos gefint zikh. Zayn itstiger artikel basheftigt zikh mit der atak fun a Kvibeker galakh un zayne kloymershdike tsitaten fun Talmud, vos iz geven gedrukt in der "frumer" kvibeker tsaytung "L'Action catholique". Yeder ayner vos vil visen dem emes vegen der batsyung fun Talmud tsu di umes-hoo'ylem, darf leyenen dem vikhtigen artikel fun an emese'n ekspert in talmudisher literatur". »

\section{6}

«Pour des raisons cachées - raisons qui ne peuvent être révélées - le rabbin Hirschprung a dû mettre un terme à la parution de la série. "Note de la rédaction qui précède la reprise de la parution du récit dans le Keneder Adler le 3 mai 1944.

\section{7}

«Sof yor 1942 hot horav Hirschprung ongehoyben tsu druken in Keneder Adler a serye « Zikhroynes fun a polet, » velkhe iz mit groys interest nokhgefolgt gevorn fun unzere lezers. [...] Mir zenen tsufriden tsu melden, az di interesante zikhroynes velen fun itst on umunterbrokhen forgezets veren un bikhdey di serye zol nit farliren fun ir gantskayt farefentlikhen mir dos ershte kapitel mit a kitser, fun di biz itst gedrukte kapitlen » op. cit.

\section{8}

L'auteur fait ici référence aux deux « rives » de l'océan Atlantique, comme s'il s'agissait d'une simple rivière.

\section{9}

Ce nom inhabituel « celui qui donne des conseils » était le pseudonyme employé par Haim Kruger pour faire valoir ses talents de conteur et de conseiller domestique. 
30

«Portraits intimes et mémoires au sujet de figures juives connues en Pologne. » Malgré son titre, ce feuilleton de Melekh Ravitch n'avait rien à voir avec le conflit militaire en cours.

\section{1}

L'ouvrage traduit en anglais par A. M. Klein, a paru en 1945 sous le titre Journey of My Life, et en version originale yiddish en 1946. Une version française a été publiée en 2000 par l'auteur de cet article sous le titre: Mayn lebens rayze; un demi-siècle de vie yiddish à Montréal. L'auteur ne fait aucune mention directe à la Seconde Guerre mondiale dans ce texte.

\section{2}

Voir par exemple « Hurbn beys Yisroel [la destruction de la maison d'Israël], » publié le 10 décembre 1943, «Shrites [massacres], » paru le 17 mars 1944 et « Ze velen zikh nit rateven [ils ne survivront pas], » publié le 28 avril 1944.

\section{3}

Voir Pierre Anctil, Jacob-Isaac Segal (18961954), un poète yiddish de Montréal et son milieu (Québec, les Presses de l'Université Laval, 2012).

\section{4}

«Iz es deriber nit kayn « literatur » ot dos vos er dertsaylt unz fun zayne iberlebungen. S'iz der emes alayn. [...] Ikh loz es iber tsu di kritiker tsu urtaylen vegen der literarisher zayt fun dem bukh. Ikh fun mayn zayt fil bloyz vi tsu bagrisen di dershaynung fun ot der sefer, vi a faynem menshlikhen dokument. »

\section{5}

Franklin Bialystok, Delayed Impact: the Holocaust and the Canadian Jewish Community (Montreal, McGill-Queen's University Press, 2000). 